\title{
Liraglutide ameliorates glycometabolism and insulin resistance through the upregulation of GLUT4 in diabetic KKAy mice
}

\author{
LI-NA CHEN ${ }^{1,3}$, JUAN LYU $^{1,3}$, XUE-FEI YANG ${ }^{1,3}$, WEN-JUN JI ${ }^{1,3}$, BING-XIANG YUAN ${ }^{1,3}$, \\ MING-XIA CHEN ${ }^{2}, \mathrm{XIN} \mathrm{MA}^{1}$ and BING WANG ${ }^{1,3}$ \\ ${ }^{1}$ Department of Pharmacology and ${ }^{2}$ Center of Electron Microscopy, College of Medicine, Xi'an Jiaotong University; \\ ${ }^{3}$ Therapeutic Vaccine Research Center of Shaanxi Province, Xi'an, Shaanxi 710061, P.R. China
}

Received April 9, 2013; Accepted July 12, 2013

DOI: $10.3892 /$ ijmm.2013.1453

\begin{abstract}
Liraglutide, a long-lasting glucagon-like peptide-1 analogue, has been used for the treatment of patients with type 2 diabetes mellitus since 2009. In this study, we investigated the anti-diabetic effects and mechanisms of action of liraglutide in a spontaneous diabetic animal model, using KK/Upj-Ay/J (KKAy) mice. The KKAy mice were divided into 2 groups, the liraglutide group (mice were treated with $250 \mu \mathrm{g} / \mathrm{kg} / \mathrm{day}$ liraglutide) and the model group (treated with an equivalent amount of normal saline). C57BL/6J mice were used as the controls (treated with an equivalent amount of normal saline). The treatment period lasted 6 weeks. During this treatment period, fasting blood glucose (FBG) levels and the body weight of the mice were measured on a weekly basis. Our results revealed that liraglutide significantly decreased FBG levels, the area under the curve following a oral glucose tolerance test and insulin tolerance test, increased serum insulin levels, reduced homeostasis model assessment of insulin resistance and increased the insulin sensitivity index. Furthermore, liraglutide ameliorated glycometabolism dysfunction by increasing glycolysis via hexokinase and glycogenesis via pyruvate kinase activation. An ultrastructural examination of the pancreas revealed that liraglutide improved the damaged state of islet $\beta$ cells and increased the number of insulin secretory granules. The real-time PCR results revealed that the gene expression
\end{abstract}

Correspondence to: Dr Bing Wang, Department of Pharmacology, College of Medicine, Xi'an Jiaotong University, No. 76 Yanta West Road, Xi'an, Shaanxi 710061, P.R. China

E-mail:wangbing@mail.xjtu.edu.cn

Abbreviations: FBG, fasting blood glucose; AUC, area under the curve; OGTT, oral glucose tolerance test; ITT, insulin tolerance test; HOMA-IR, homeostasis model assessment of insulin resistance; ISI, insulin sensitivity index; HK, hexokinase; PK, pyruvate kinase; GLP-1, glucagon-like peptide-1; InsR, insulin receptor; PI3K, phosphatidylinositide 3-kinase; GLUT, glucose transporter; KKAy, KK/Upj-Ay/J; C57, C57BL/6J

Key words: liraglutide, glycometabolism, insulin resistance, glucose transporter 4, KK/Upj-Ay/J mice of glucose transporter 4 (GLUT4) increased following treatment with liraglutide. Liraglutide also upregulated the protein expression of GLUT4 in liver tissue and skeletal muscle. Our results suggest that liraglutide ameliorates glycometabolism and insulin resistance in diabetic KKAy mice by stimulating insulin secretion, increasing glycogenesis and glycolysis and upregulating the expression of GLUT4.

\section{Introduction}

It has been reported that approximately 366 million individuals worldwide were affected by diabetes in 2011 and the numbers are estimated to reach 522 million by 2030 (1). With the increase in diabetes cases, complications associated with diabetes, such as large-vessel obstruction (including coronary artery diseases, atherosclerosis and peripheral vascular diseases) and microvascular pathologies (including retinopathy, nephropathy and neuropathy) are also expected to increase (2). Insulin resistance is known to play a vital role in diabetes and its complications $(3,4)$.

Glucagon-like peptide-1 (GLP-1), an anorexigenic hormone, secreted by intestinal L-cells, has several physiological functions, such as lowering blood glucose levels and increasing insulin secretion (5-7). Liraglutide, marketed under the brand name, Victoza, is a long-acting GLP-1 agonist developed by Novo Nordisk (Bagsvaerd, Denmark) for the treatment of type 2 diabetes (8). The product was approved by the European Medicines Agency (EMA) on July 3, 2009 and by the US Food and Drug Administration (FDA) on January 25, 2010. It has been reported that liraglutide activates the GLP-1 receptor and exerts several physiological effects, such as decreasing blood glucose levels, improving lipid metabolism and reducing blood pressure and endothelial dysfunction $(9,10)$. However, the mechanisms behind its anti-diabetic effects remain unknown.

Studies have confirmed that important insulin signaling molecules, such as insulin receptor(InsR), phosphatidylinositide 3-kinases (PI3Ks) and glucose transporter (GLUT), play a vital role in glucose metabolism and insulin resistance $(11,12)$. In particular, GLUT4 is one of the predominant GLUTs in cells, promoting glucose trafficking and uptake. The concentration of glucose increases abnormally with anomalies in GLUT4 expression $(13,14)$. KK/Upj-Ay/J (KKAy) mice can spontaneously become obese, hyperglycemic and hypertriglyceridemic, 
and are thus used as a polygenic type 2 diabetes model (15). In the present study, we used diabetic KKAy mice to investigate the effects and the mechanisms of action of liraglutide on glucose metabolism and insulin resistance.

\section{Materials and methods}

Animals, grouping and treatment. Twelve male KKAy mice (11-13 weeks old) and 6 C57BL/6J (C57) mice (same age) were obtained from the Chinese Academy of Medical Sciences (Beijing, China) and were kept individually in plastic cages covered with wood shavings at a temperature of $20-25^{\circ} \mathrm{C}$ and a humidity of $45-55 \%$ with illumination for $12 \mathrm{~h}$ in a specific pathogen-free (SPF) environment. The KKAy mice were fed high-fat chow and the C57 mice were fed ordinary animal chow. All the mice were allowed free access to water. The present study was approved by the Ethics Committee on the Use of Experimental Animals of Xi'an Jiaotong University, Xi'an, China.

After the mice were allowed to acclimatize for 1 week, fasting blood glucose (FBG) levels in the KKAy mice were measured. The mice with FBG values $>16.7 \mathrm{mmol} / 1$ were considered diabetic and randomly divided into 2 experimental groups: the liraglutide group [n=6, subcutaneous (s.c.) liraglutide injection $250 \mu \mathrm{g} / \mathrm{kg} /$ day; obtained from Novo Nordisk] and the model group $(n=6$, s.c. equivalent volume of normal saline). The C57 mice were considered non-diabetic and used as the control group ( $n=6$, s.c. equivalent volume of normal saline). Drugs were administered to the mice between 16:30 and 17:00 p.m. each day. During the 6-week treatment period, FBG levels (6-h fast) and body weights of all the animals were measured weekly using a glucose meter (Roche Diagnostics $\mathrm{GmbH}$, Mannheim, Germany) and a laboratory electronic scale. At the end of the experiment, blood samples from the KKAy mice were obtained through the venous plexus behind the eyeball following anesthesia by $10 \%$ chloral hydrate. The samples were placed into Eppendorf tubes and centrifuged at $1,368 \mathrm{x} \mathrm{g}$ for $10 \mathrm{~min}$ at room temperature and the plasma was collected and stored at $-80^{\circ} \mathrm{C}$ until analysis. The mice were then sacrificed. Tissues were separated into 3 specimens with one immediately stored in Eppendorf tubes frozen in liquid nitrogen and the second immersed into stationary liquid and observed under an electron microscope and then rapidly placed in a refrigerator at $4^{\circ} \mathrm{C}$ until analysis. The third was fixed in $10 \%$ formalin for immunohistochemical examination.

Oral glucose tolerance test (OGTT). The OGTT was performed on the 7th week of the experimental period. After $6 \mathrm{~h}$ of fasting, a glucose solution of $2 \mathrm{~g} / \mathrm{kg}$ was orally administered, and blood glucose was measured at 0,30,60 and $120 \mathrm{~min}$, after obtaining blood from an injection into the tail vein, using a glucose meter and the area under the curve (AUC) was calculated for FBG during the OGTT.

Insulin tolerance test (ITT). After 6 weeks of treatment, an ITT was performed. A subcutaneous injection of $4 \mathrm{U} / \mathrm{kg}$ insulin (Jiangsu Wanbang Biochemistry Medicine Co., Xuzhou, China) was administered after $6 \mathrm{~h}$ of fasting and blood glucose levels were measured at 0,40 and 90 min using a glucose meter. The AUC was calculated for FBG during the ITT.
Serum insulin detection and insulin resistance index calculation. Serum insulin levels were measured by enzyme-linked immunosorbent assay (ELISA) using a mouse insulin ELISA kit (R\&D System, Inc., Minneapolis, MN, USA). The homeostasis model assessment of insulin resistance (HOMA-IR) and the insulin sensitivity index (ISI) were calculated according to the following formula: HOMA-IR = FBG $(\mathrm{mmol} / \mathrm{l}) \times$ FINS $(\mu \mathrm{U} / \mathrm{ml}) / 22.5 ; \mathrm{ISI}=1 /[\mathrm{FBG}(\mathrm{mmol} / \mathrm{l}) \times$ FINS $(\mu \mathrm{U} / \mathrm{ml})]$, where FINS represents the fasting insulin levels, as previously described (16).

Liver and skeletal muscle biochemical index analysis. Liver and skeletal muscle glycogen levels were measured using the anthracenone method. Skeletal muscle pyruvate kinase (PK) levels were measured by colorimetric assay and hexokinase (HK) levels were measured using the glucose 6 phosphate dehydrogenase coupling colorimetric method. The measurements of such indexes were performed using commercial kits (Nanjing Jiancheng Bioengineering Institute, Nanjing, China).

Transmission electron microscopy. For electron microscopy, portions of the splenic region of each pancreas were minced into $1-\mathrm{mm}$ cubes, fixed with $2.5 \%$ glutaraldehyde and $2 \%$ osmic acid and then dehydrated and embedded in epoxy resin. Ultrathin sections were collected onto 200-mesh copper grids and double stained with uranyl acetate and lead acetate, and each section was observed under a Hitachi H-7650 Transmission Electron Microscope (Hitachi, Tokyo, Japan).

RNA isolation and real-time PCR. Total RNA was isolated from mouse liver tissues using TriPure RNA isolation reagent (Roche, Basel, Switzerland), and $2 \mu \mathrm{g}$ of RNA were reversetranscribed using the Prime Script ${ }^{\mathrm{TM}}$ RT Master Mix (Perfect Real Time) (Takara Bio, Inc., Tokyo, Japan). Quantitative RT-PCR was performed using SYBR ${ }^{\circledR}$ Premix Ex Taq $^{\text {TM }}$ II (Perfect Real-Time; Takara Bio, Inc.). PCR reactions were performed in 96-well plates in an iQ5 Real-Time PCR Detection System (Bio-Rad Laboratories, Hercules, CA, USA).

All of the primers and probes for RT-PCR were obtained from Takara Bio, Inc. Murine InsR was amplified using the primers InsR-F (5'-GCC GCT CCT ATG CTC TGG TAT C-3') and InsR-R (5'-AGT TGC CTC AGG TTC TGG TTG TC-3'); murine $\mathrm{PI} 3 \mathrm{~K}$ was amplified using the primers $\mathrm{PI} 3 \mathrm{~K}$ regulatory subunit 1 (Pik3r1)-F (5'-GCT CCT GGA AGC CAT TGA GAA-3') and Pik3r1-R (5'-CGT CGA TCA TCT CCA AGT CCA C-3'); murine GLUT2 was amplified using the primers solute carrier family 2 (facilitated glucose transporter), member 2 (Slc2a2)-F (5'-GGC ATC AGC CAG CCT GTG TA-3') and Slc2a2-R (5'-CAT GCC AAT CAT CCC GGT TAG-3'); murine GLUT4 was amplified using the primers solute carrier family 2 (facilitated glucose transporter), member 4 (Slc2a4)-F (5'-TCT TAT TGC AGC GCC TGA GTC-3') and Slc2a4-R (5'-GCC AAG CAC AGC TGA GAA TAC A-3'); and murine GAPDH was amplified using the primers GAPDH-F (5'-TGT GTC CGT CGT GGA TCT GA-3') and GAPDH-R (5'-TTG CTG TTG AAG TCG CAG GAG-3'). GAPDH served as the endogenous control. The results were normalized to GAPDH. Efficiencies of RT-PCR for the target gene and the endogenous control were approximately equal. The $-\Delta C T$ expresses the difference between the number of 

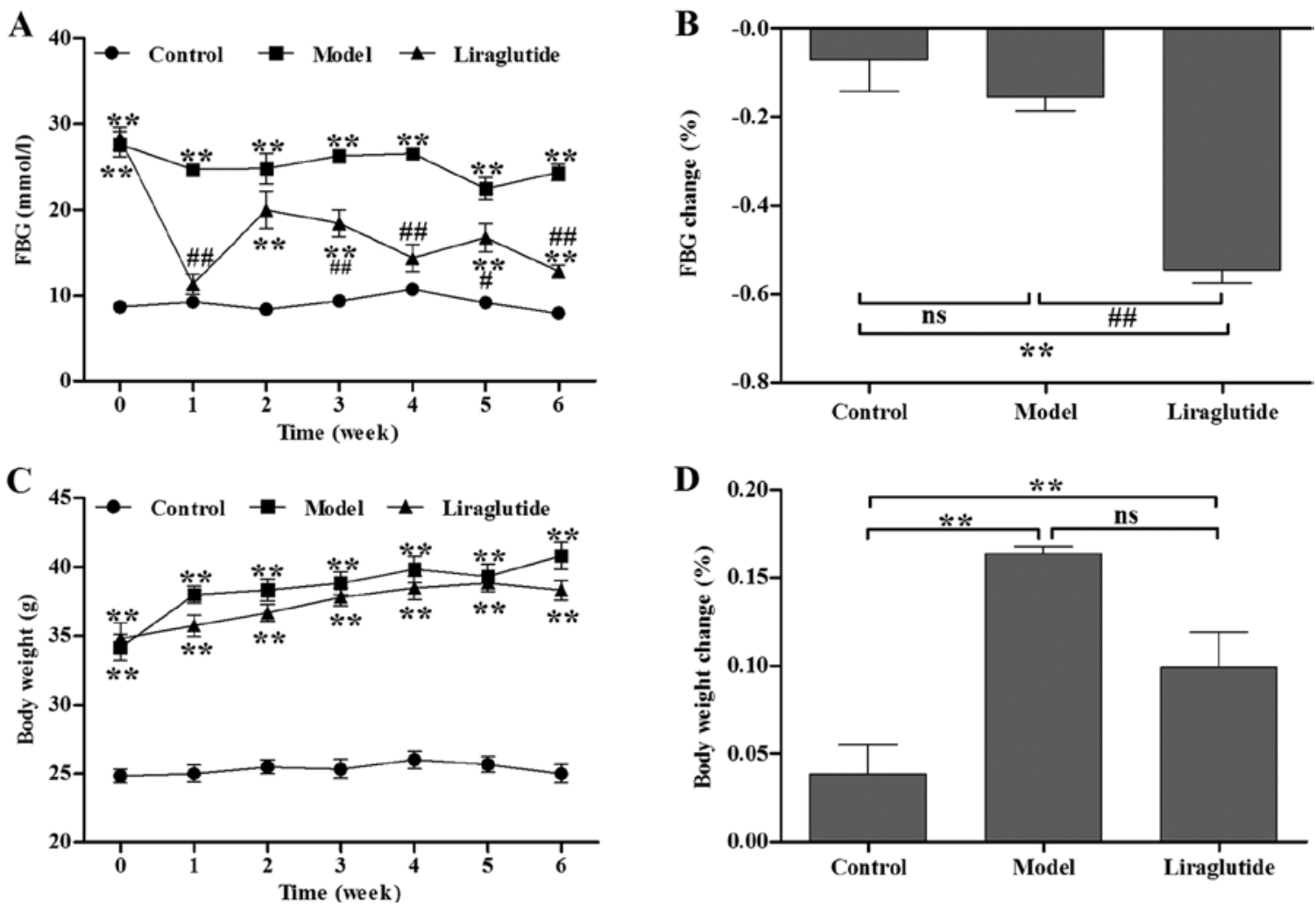

Figure 1. Effects of liraglutide on fasting blood glucose (FBG) levels and body weight in KKAy mice. (A) Weekly variation of FBG levels in KKAy mice. (B) The FBG decreasing rate in KKAy mice after treatment. (C) Changes in body weight in KKAy mice. (D) Body weight increasing rate in KKAy mice after treatment. ${ }^{* *} \mathrm{P}<0.01$, vs. control group; ${ }^{\#} \mathrm{P}<0.05,{ }^{\# \#} \mathrm{P}<0.01$, vs. model group; $\mathrm{ns}$, not significant ( $\mathrm{n}=6 /$ group).

cycles (CT) of the target genes and the endogenous control. The results were expressed as $2^{-\Delta \Delta \mathrm{Ct}}$, and express the $\mathrm{x}$-fold increase of gene expression compared with the control group. The standard curve and data analysis were produced using Bio-Rad iQ5 software (Bio-Rad Laboratories).

Immunohistochemistry (IHC) in liver tissue and skeletal muscle. IHC was performed to examine the expression of GLUT4. Liver tissue and skeletal muscle from each group were fixed in $10 \%$ formalin and embedded in paraffin. The sections were deparaffinized and rehydrated and then immersed in citrate buffer and heated in a microwave oven for antigen retrieval. After cooling to room temperature, the sections were rinsed and incubated with $3 \% \mathrm{H}_{2} \mathrm{O}_{2}$ solution to block endogenous enzymes. Normal goat serum was incubated to block non-specific binding after rinsing with PBS. Primary antibody to GLUT4 (1:200; Abcam, Cambridge, MA, USA) was then added, followed by overnight incubation at $4^{\circ} \mathrm{C}$. The sections were incubated with the appropriate secondary antibody and horseradish-peroxidase. DAB substrate buffer (Beijing Zhongshan Golden Bridge Biotechnology Co., Beijing, China) was then added to achieve coloration. The specimens were subsequently dehydrated with ethanol, cleared with xylene and then mounted on glass cover slips. Brown colored sites were quantified at a final magnification of $\mathrm{x} 400$ using a microscope by a pathologist and photographed using an Olympus DP71 digital camera (Olympus, Tokyo, Japan).

Statistical analysis. Values are presented as the means \pm standard error of the mean (SEM). Differences between the C57 mice and KKAy mice were tested for significance with an independent sample t-test and ANOVA. All statistical analyses were performed using SPSS v13.0 software and the data in the figures were analyzed using GraphPad Prism 5.0 software. Parameters with P-values $<0.05$ were considered to indicate statistically significant differences.

\section{Results}

Liraglutide decreases FBG levels and body weight in KKAy mice. Initially, baseline FBG levels in the diabetic KKAy mice were significantly higher than those in the C57 mice (Fig. 1A). Following 1 week of injections, the liraglutide-treated KKAy mice demonstrated a significant decrease in FBG levels compared with the normal saline-treated KKAy mice (Fig. 1A). During the 6 weeks of treatment, the blood glucose levels in the liraglutide group had a decreasing tendency. The decreasing percentage of FBG in the liragutide-treated KKAy mice was significantly higher than the normal saline-treated KKAy mice after the treatment period ended $(\mathrm{P}<0.01$, vs. model group) (Fig. 1B). There was no statistically significant difference in body weight between the liraglutide-treated and normal saline-treated KKAy mice before and during the treatment period ( $\mathrm{P}>0.05)$ (Fig. $1 \mathrm{C}$ and $\mathrm{D})$.

Liraglutide improves the glucose tolerance of KKAy mice. The OGTT results revealed that the blood glucose levels in all groups peaked at $30 \mathrm{~min}$ after oral glucose loading and then decreased. We observed that the blood glucose levels in the liraglutide group increased and decreased (Fig. 2A) 
A
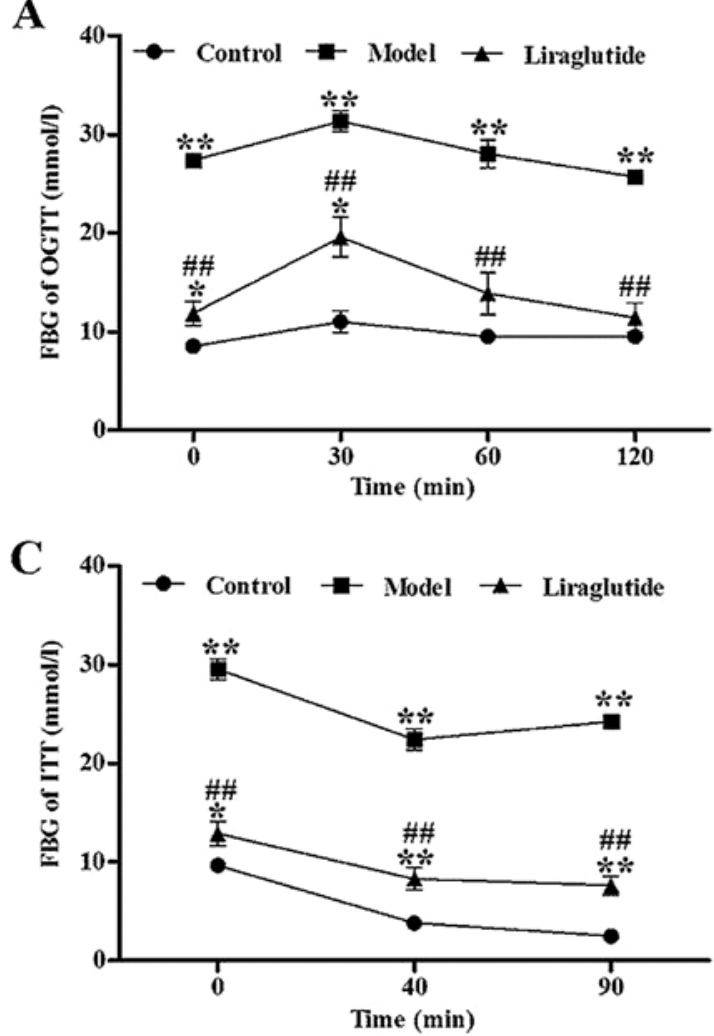

E

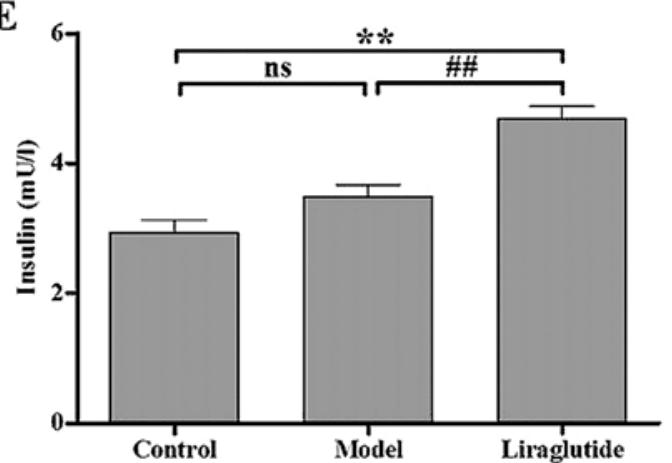

G

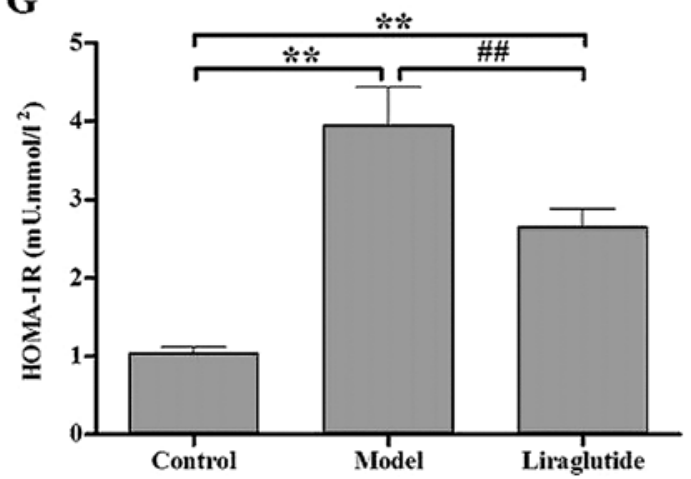

B

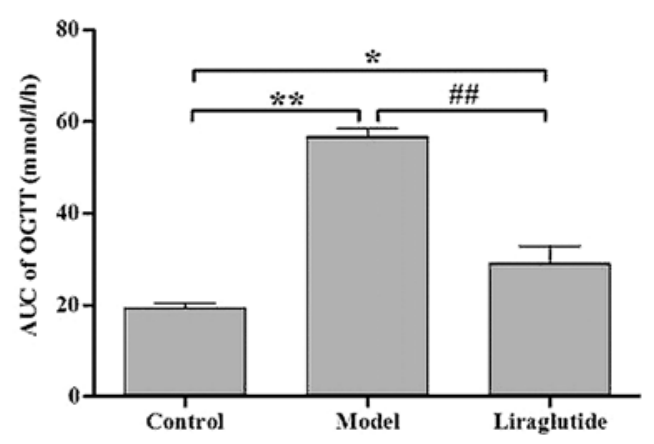

D

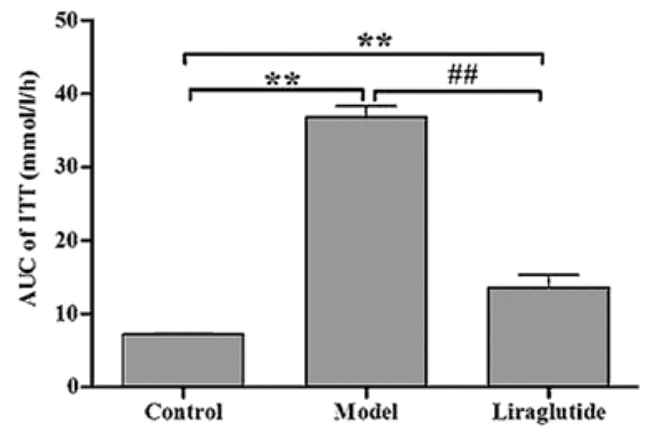

F

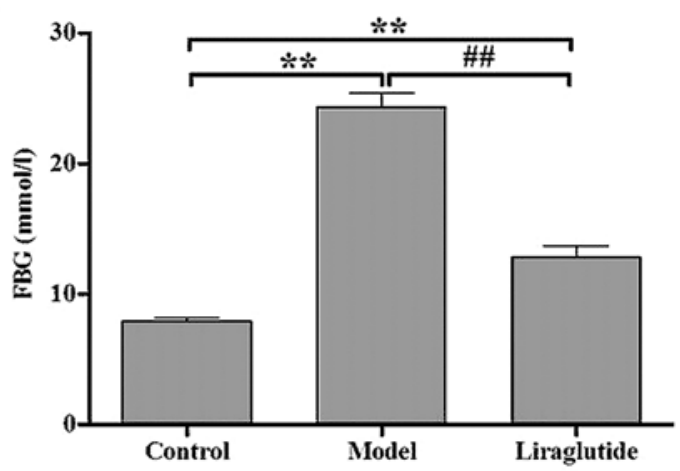

H

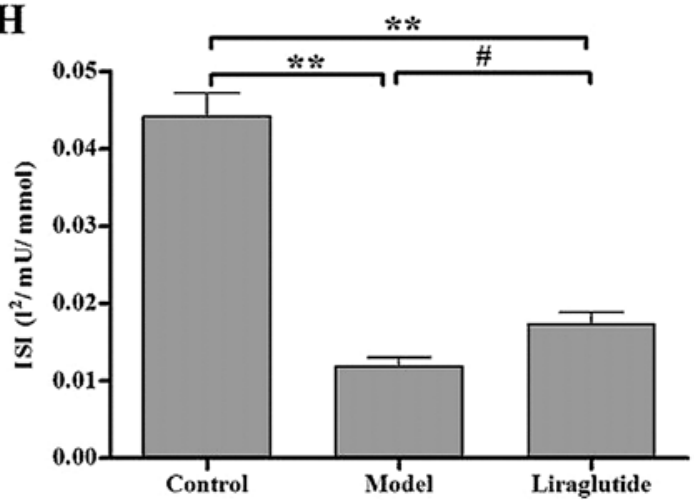

Figure 2. Effects of liraglutide on the index of tolerance and insulin resistance in KKAy mice. (A) Oral glucose tolerance test (OGTT); (B) area under the curve (AUC) of OGTT; (C) insulin tolerance test (ITT); (D) AUC of ITT; (E) serum insulin levels; (F) fasting blood glucose (FBG); (G) homeostasis model assessment of insulin resistance (HOMA-IR); (H) insulin sensitivity index (ISI). ${ }^{*} \mathrm{P}<0.05,{ }^{* *} \mathrm{P}<0.01$, vs. control group; ${ }^{\#} \mathrm{P}<0.05$, ${ }^{\# \#} \mathrm{P}<0.01$, vs. model group; ns, not significant ( $\mathrm{n}=6 /$ group).

much more promptly in a persistent state compared with the control and model group, whereas the levels of blood glucose in the model group remained at high levels before and after the glucose solution loading. The AUC in the liraglutide group was significantly lower than that in the model group $(\mathrm{P}<0.01)$ (Fig. 2B). The AUC in the liraglutide group was significantly 
A
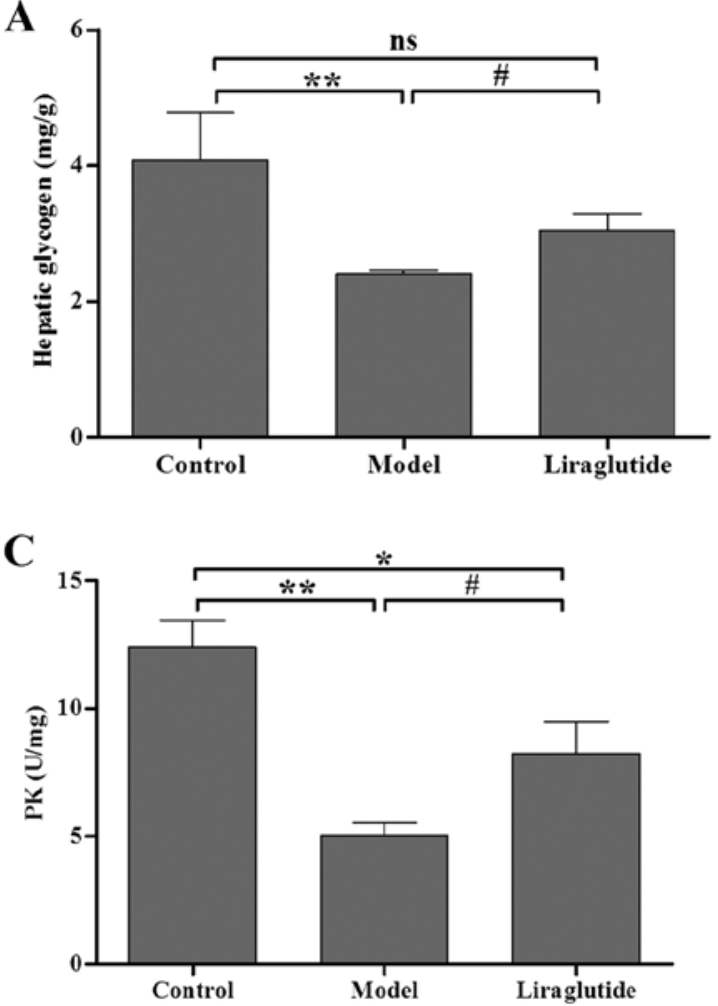

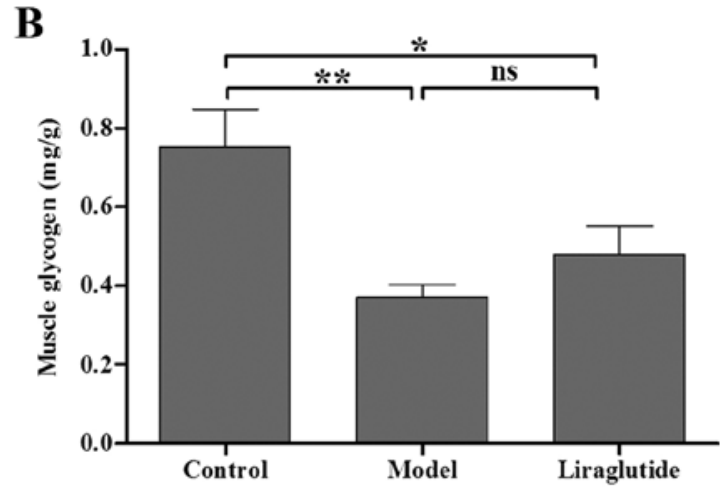

D

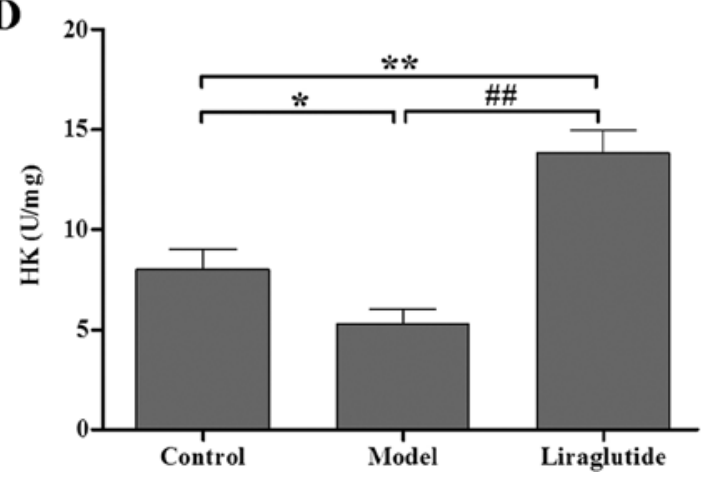

Figure 3. Effect of liraglutide on glycometabolism biochemical parameters. (A) Effect of liraglutide on the increased hepatic glycogen content. (B) Effect of liraglutide on the increased skeletal muscle glycogen content. (C) Liraglutide increased skeletal muscle pyruvate kinase activity. (D) Liraglutide increased skeletal muscle hexokinase activity. ${ }^{*} \mathrm{P}<0.05,{ }^{* *} \mathrm{P}<0.01$, vs. control group; ${ }^{\#} \mathrm{P}<0.05,{ }^{\# \#} \mathrm{P}<0.01$, vs. model group; ns, not significant (n=6/group). PK, pyruvate kinase; HK, hexokinase.

higher than that in the control group $(\mathrm{P}<0.05)$ (Fig. 2B). These results demonstrate that liraglutide improves glucose tolerance in KKAy mice.

Liraglutide improves insulin tolerance in KKAy mice. In all the groups, FBG levels rapidly decreased within $40 \mathrm{~min}$ after the insulin injection. In the control group and liraglutide group, the FBG levels continued to decrease (Fig. 2C). However, the blood glucose levels in the model group began to gradullay increase after $40 \mathrm{~min}$. The AUC in the liraglutide group was significantly lower than that in the model group $(\mathrm{P}<0.05)$ (Fig. 2D) and higher than that in the control group $(\mathrm{P}<0.05)$ (Fig. 2D). These results indicate that liraglutide ameliorates insulin tolerance in KKAy mice.

Liraglutide improves serum biochemical parameters in KKAy mice. Following treatment with liraglutide for 6 weeks, the insulin levels in the liraglutide group were significantly higher than those in the control and model group $(\mathrm{P}<0.01)$ (Fig. 2E). Fig. $2 \mathrm{~F}$ illustrates the FBG levels of the 3 groups at the end of the experiment. HOMA-IR in the liraglutide group was significantly decreased compared with the model group $(\mathrm{P}<0.05)$, but was still higher compared with the control group $(\mathrm{P}<0.05)$ (Fig. 2G). Likewise, the ISI in the liraglutide group significantly improved compared with the model group $(\mathrm{P}<0.05)$, but was still higher compared with the control group $(\mathrm{P}<0.05)($ Fig. $2 \mathrm{H})$.

Liraglutide influences the glucometabolic biochemical index in KKAy mice. As indicated by the content of hepatic glycogen, liraglutide significantly increased glucose metabolism in the liver compared with the normal saline-treated KKAy mice $(\mathrm{P}<0.05)$ (Fig. 3A). As indicated by the levels of skeletal muscle glycogen, following treatment with liraglutide, glucose metabolism in skeletal muscle improved; however, no difference was observed between the model group and the liraglutide group (P>0.05) (Fig. 3B). As indicated by the skeletal muscle PK and HK levels, liraglutide significantly increased PK and HK activity compared with the model group $(\mathrm{P}<0.05)$ (Fig. 3C and D). In the normal C57 mice, skeletal muscle PK and HK levels were significantly higher than those of the KKAy mice $(\mathrm{P}<0.05)$ (Fig. 3C and D).

Effects of liraglutide on ultrastructure of $\beta$ cells. Ultrastructural changes were observed in the pancreatic $\beta$ cells of the KKAy mice compared with the C57 mice (Fig. 4). The number of secretory granules, which presumably contain insulin, in the $\beta$ cells from the normal saline-treated KKAy mice was found to be markedly lower than that in the control C57 mice. Enlargement of the mitochondria, destruction of mitochondrial cristae, development of the Golgi apparatus and an increase in the amount of the rough endoplasmic reticulum (RER) in the electron microscopic images of pancreatic $\beta$ cells from the normal saline-treated KKAy mice were observed compared with the C57 mice. The number of secretory granules increased in the $\beta$ cells from the liraglutide-treated KKAy mice; the morphology of the mitochondria, the Golgi apparatus and the RER had a more normal appearance, comparable to that in C57 mice.

Effects of liraglutide on gene expression in KKAy mice. As shown in Fig. 5, the expression of all the selected target genes 

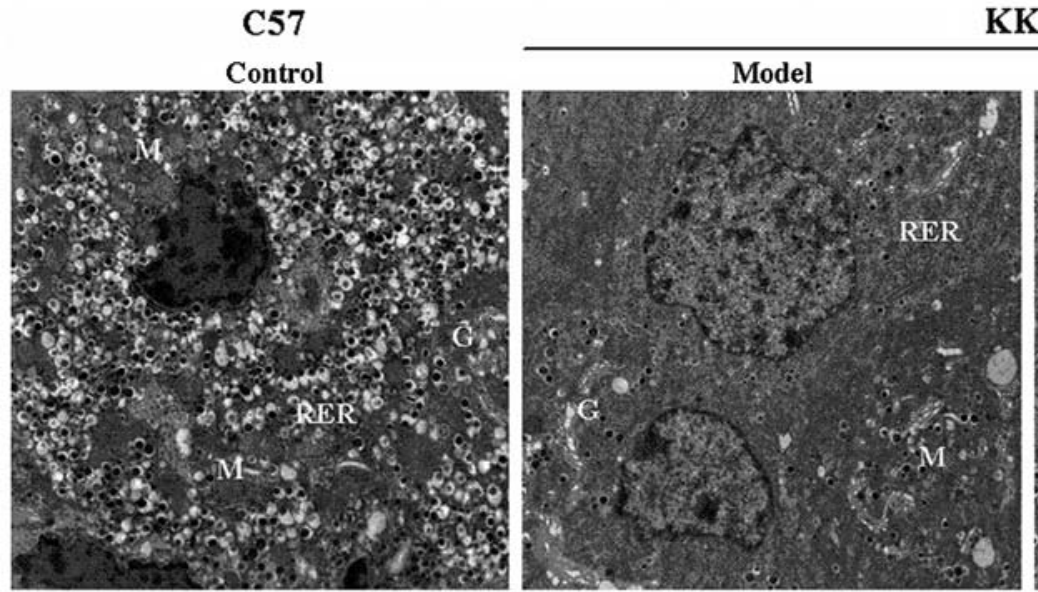

\section{KKAy}

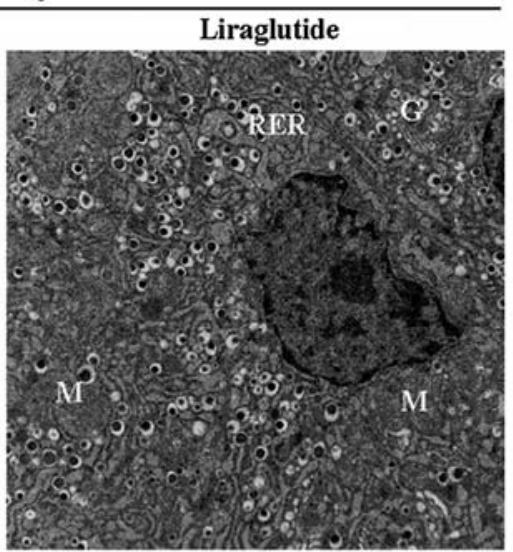

Figure 4. Effect of liraglutide on ultrastructure of pancreatic $\beta$ cells. Liraglutide ( $250 \mu \mathrm{g} / \mathrm{kg} / \mathrm{day})$ or normal saline was administered daily to KKAy mice for 6 weeks. At the end of treatment, pancreatic tissues were excised and fixed with $2.5 \%$ glutaraldehyde and $2 \%$ osmic acid, then dehydrated and embedded in epoxy resin. Ultra-thin sections were stained with uranyl acetate and lead acetate. Each section was observed under an electron microscope. Representative images of pancreatic $\beta$ cell sections of age-matched normal saline-treated C57 mice, normal saline-treated KKAy mice, or liraglutide-treated KKAy mice are shown (x10,000). G, Golgi apparatus; M, mitochondria; RER, rough endoplasmic reticulum.

A

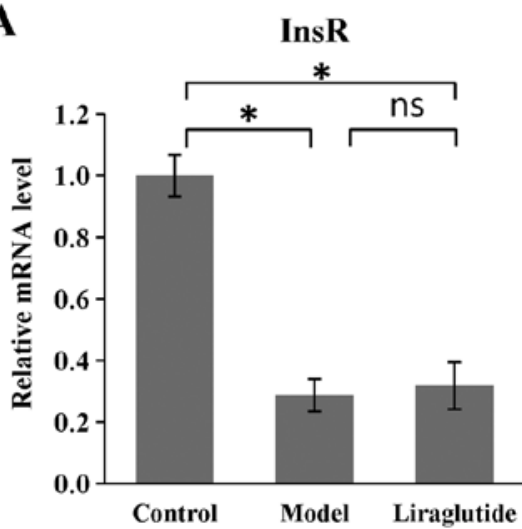

C

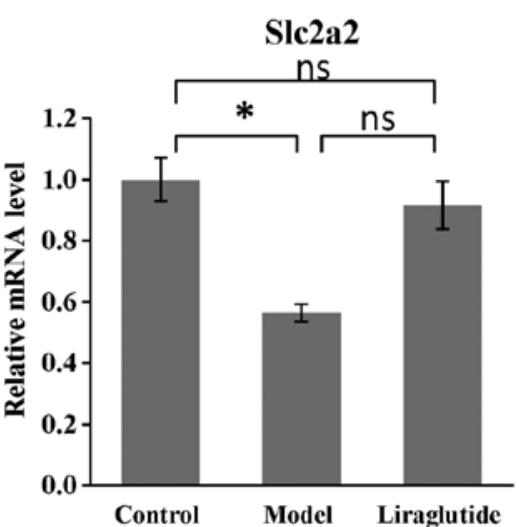

B

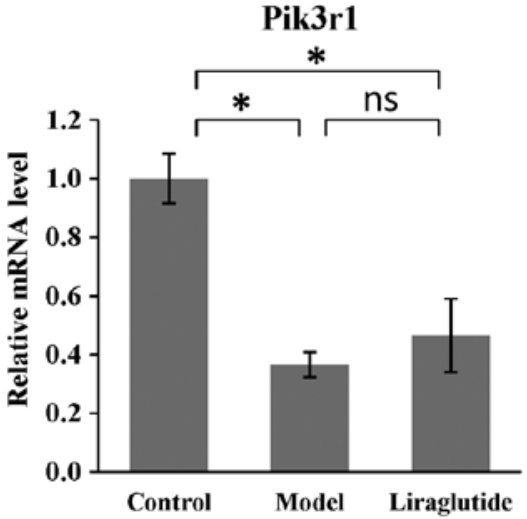

D

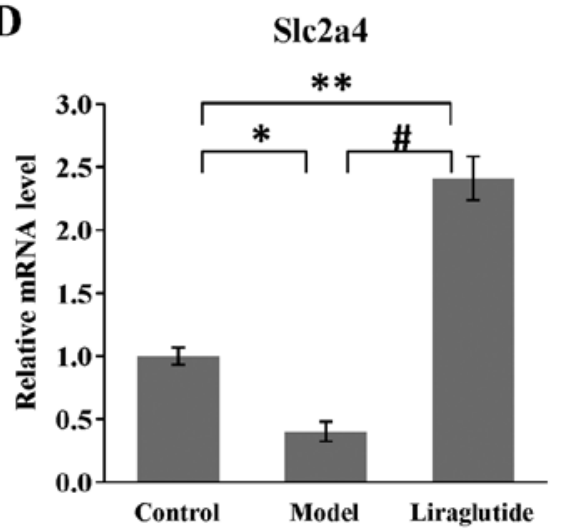

Figure 5. Effect of liraglutide on gene expression in liver tissue of C57 mice and KKAy mice. (A) Insulin receptor (InsR), (B) phosphatidylinositide 3-kinase regulatory subunit 1 (Pik3r1), (C) solute carrier family 2 (facilitated glucose transporter), member 2 (Slc2a2) (coding GLUT2) and (D) solute carrier family 2 (facilitated glucose transporter), member 4 (Slc2a4) (coding GLUT4). The control group comprised C57 mice treated with normal saline; the model group and liraglutide group were KKAy mice treated with normal saline or liraglutide for 6 weeks, respectively. ${ }^{*} \mathrm{P}<0.05,{ }^{* *} \mathrm{P}<0.01$, vs. control group; ${ }^{\sharp} \mathrm{P}<0.05$, vs. model group (n=3/group); ns, not significant.

(InsR, Pik3r1, Slc2a2, Slc2a4) in the insulin pathway was decreased in KKAy mouse livers, compared with those in the C57 mice $(\mathrm{P}<0.05$ or $\mathrm{P}<0.01)$. Following the administration of liraglutide for 6 weeks, the expression of Slc2a4 (coding GLUT4) increased significantly compared with that in the model group (KKAy mice treated with normal saline; P<0.01) (Fig. 5D). Although the other genes showed no difference in expression between the model group and liraglutide group, the expression 
A

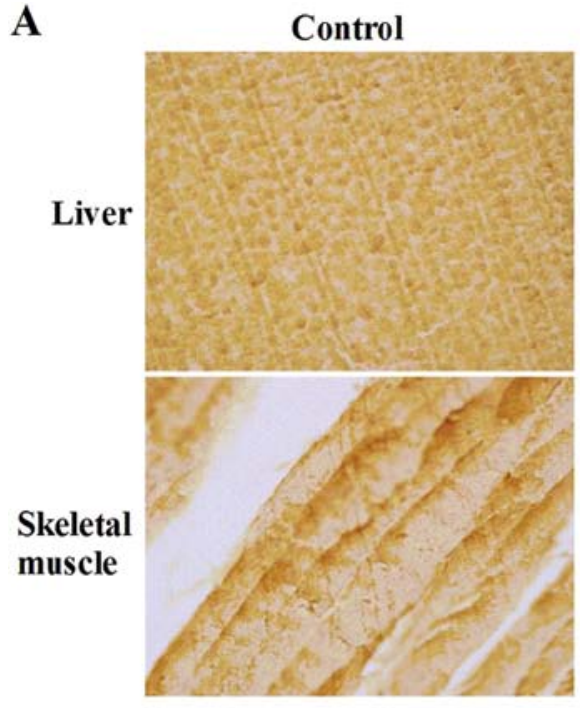

B

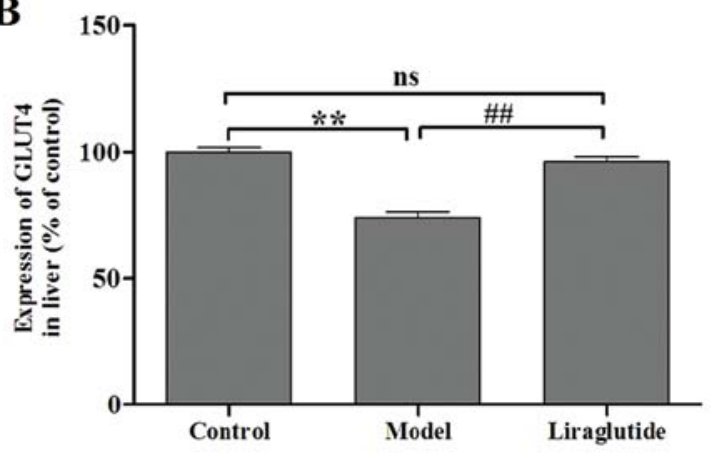

Model
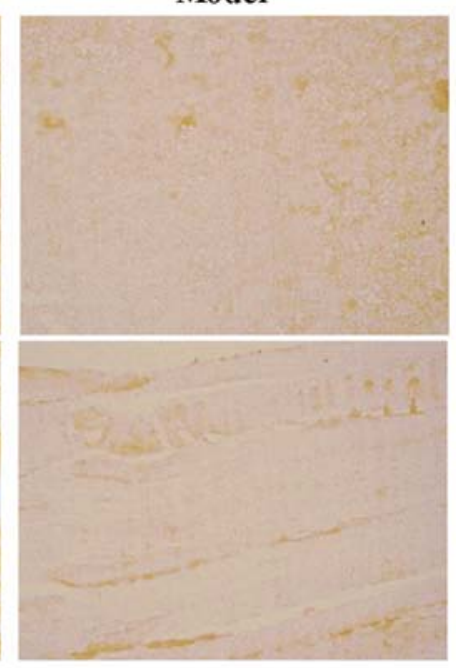

\section{Liraglutide}

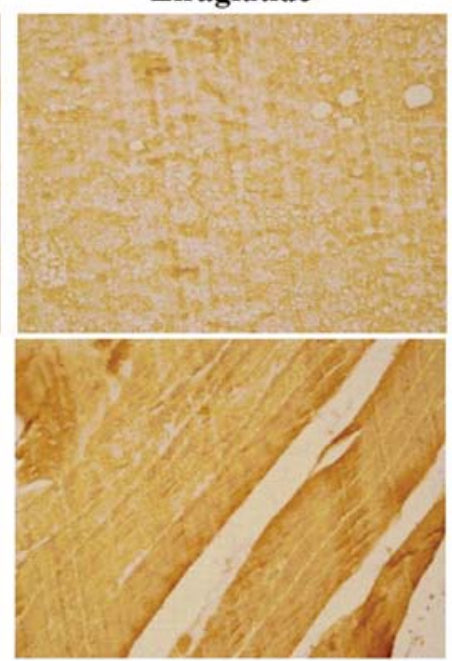

C

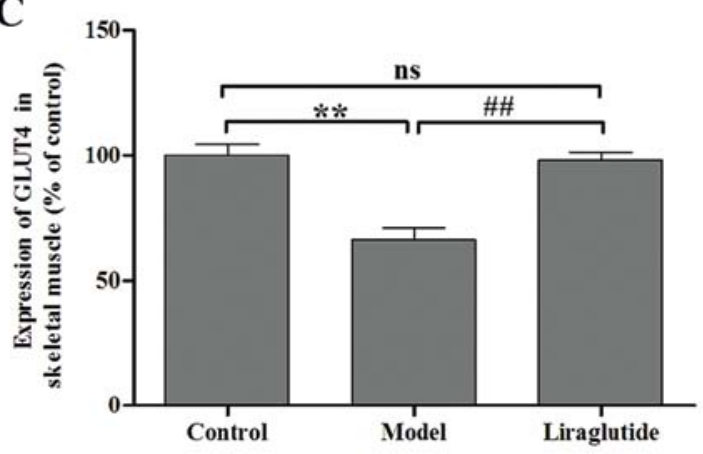

Figure 6. Immunohistochemical staining of glucose transporter 4 (GLUT4) in liver and skeletal muscle following treatment with liraglutide (250 $\mu \mathrm{g} / \mathrm{kg} / \mathrm{day})$ or normal saline for 6 weeks. At the end of treatment, liver and skeletal muscle tissues were fixed in $10 \%$ formalin for immunohistochemical examination. (A) Representative images of GLUT4 expression in the control group (normal saline-treated C57 mice), model group (normal saline-treated KKAy mice), or liraglutide-treated KKAy mice are shown (x400). (B) Liraglutide increased the expression of GLUT4 in KKAy mouse liver tissues. (C) Liraglutide increased the expression of GLUT4 in KKAy mouse skeletal muscles. ${ }^{* *} \mathrm{P}<0.01$, vs. control group; ${ }^{\# \#} \mathrm{P}<0.01$, vs. model group; ns, not significant.

of Slc2a2 (coding GLUT2) was slightly increased, which was not statistically different compared with that in the C57 mice $(\mathrm{P}>0.05)$ (Fig. 5C).

IHC in liver tissue and skeletal muscle. The positive signal of GLUT4 was presented as brown (Fig. 6). We used Image-Pro Plus 6.0 software to analyze the IHC images and found that following liraglutide administration, the expression of GLUT4 in liver tissue and skeletal muscle increased significantly compared with the model group $(\mathrm{P}<0.01)$ (Fig. 6). These results demonstrate that liraglutide upregulates the expression of GLUT4 in liver tissue and skeletal muscle in diabetic KKAy mice.

\section{Discussion}

Liraglutide, a long-lasting GLP-1 analogue, has been used for the treatment of patients with type 2 diabetes mellitus since 2009. The degree of sequence identity between liraglutide and native GLP-1 is high, at 97\%. Liraglutide is suitable for quaque die (q.d.; one a day) dosing via subcutaneous injection with an average half-life $\left(\mathrm{t}_{1 / 2}\right)$ of 11.6 to $12.8 \mathrm{~h}$, without regard to meals (17). The activation of the GLP-1 receptor on $\beta$ cells results in insulin production and exocytosis in a glucose-dependent manner (i.e., only during hyperglycemia), which minimizes the risk of hypoglycemia. However, the mechanisms behind its effects on insulin resistance and glycometabolism remain unknown. In our study, we used a spontaneous diabetic animal model (KKAy mice) to investigate the mechanisms behind the anti-diabetic effects of liraglutide. Our experiments confirmed that liraglutide decreases FBG levels, increases insulin secretion, improves insulin sensitivity and ameliorates insulin resistance in KKAy mice. Moreover, liraglutide increased glycogen in the liver and skeletal muscle and activated HK and PK. Furthermore, liraglutide improved $\beta$ cell morphology, increased insulin secretion, enhanced the gene expression of GLUT4 in the liver and upregulated the expression of GLUT4 in liver tissue and skeletal muscle in diabetic KKAy mice.

Several studies have reported that GLP-1 receptor agonists (e.g., exenatide and liraglutide) stimulate the production and secretion of insulin from pancreatic $\beta$ cells, reduce plasma glucose levels and reduce gastric emptying in patients with type 2 diabetes mellitus and rodents, such as Zucker Diabetic Fatty (ZDF) rats, Otsuka-Long-Evans-Tokushima Fatty (OLETF) rats, db/db mice, ob/ob mice, Swiss TO mice and $\beta$ cell-specific glucokinase-deficient Gck(-/-) mice (18-20). 
KKAy mice recapitulate the characteristics of human type 2 diabetes mellitus, such as insulin resistance, hyperglycemia and obesity (15), as a spontaneous type 2 diabetic model. Our present results demonstrate that treatment with liraglutide reduces FBG levels, lowers the AUC following OGTT and ITT, lowers the HOMA-IR index, increases insulin levels, ameliorates glucose tolerance and insulin sensitivity in diabetic KKAy mice, which are consistent with the results from the abovementioned studies (18-20).

It is well known that glucose homeostasis disorder and hyperglycemia and diabetes are closely related. Glycogen, HK and PK are important glycometabolism indexes that correlate with glucose distribution and uptake (21). It has been reported that glycogen synthesis disorder promotes the occurrence of hyperglycemia and diabetes $(22,23)$. HK and PK are the key enzymes of glycolysis. HK catalyzes glucose to generate glucose 6 phosphate in the first step in glycolysis. Activated HK promotes glycogen synthesis and glycolysis $(24,25)$. PK catalyzes the conversion of phosphoenolpyruvate to pyruvic acid and the phosphorylation of adenosine diphosphate (ADP) to adenosine triphosphate (ATP) $(26,27)$. Sun et al (28) and Ye et al (29) found that the increased expression of PK M2 (one of the PK isoforms) stimulates glycolysis. Shirakawa et al (30) reported that liraglutide increases the glycogen content in neonatal Gck(-/-) mouse livers. Our results firstly demonstrated that liraglutide increases skeletal muscle HK and PK activity. We also demonstrated that liraglutide increased liver and skeletal muscle glycogen content in KKAy mice.

Islet $\beta$ cell dysfunction and insulin resistance are the main risk factors correlated with type 2 diabetes $(31,32)$. Islet $\beta$ cell dysfunction induces insulin secretion disorders and hyperglycemia $(33,34)$. The study by Emamaullee et al $(35)$ confirmed that liraglutide improves $\beta$ cell function by reducing apoptosis. Sturis et al (36) treated ZDF rats with liraglutide for 6 weeks and found that liraglutide induced islet $\beta$ cell proliferation, thus inducing anti-hyperglycemic effects. The study by Knudsen (37) demonstrated that liraglutide modulates the progressive loss of $\beta$ cell function that drives the continous deterioration in glycaemic control in patients with type 2 diabetes. These findings are in accordance with our electron microscopy observations that liraglutide normalized the state and number of mitochondria, the Golgi apparatus and the RER, and increased the number of secretory granules in islet $\beta$ cells. The serum content of insulin was also increased by liraglutide in KKAy mice, which suggests that liraglutide improves $\beta$ cell morphology and function.

Diabetes mellitus is part of the insulin resistance syndrome and insulin resistance leads to a decrease in insulin sensitivity. Insulin secretion abnormalities and insulin sensitivity disorders in the target organs lead to blood glucose abnormalities $(38,39)$. Insulin is secreted by islets $\beta$ cells as a response mechanism for counteracting the increasing excess amounts of glucose in the blood. After insulin enters the bloodstream, it binds to the $\alpha$-subunit of the InsR on the cell membrane, which triggers the tyrosine kinase activity of the $\beta$-subunit that is attached to the $\alpha$-subunit. The InsR substrate family, such as IRS- 1 and IRS-2, is phosphorylated and combines with the regulatory subunit of PI3K and passes insulin signaling to the protein kinase B (Akt) molecule, leading to the induction of a variety of insulin bioactivities, thus improving insulin resistance (11,40-43).
GLUTs are a wide group of membrane proteins, one of the downstream substrates of Akt, which facilitate the transport of glucose over the plasma membrane. GLUT2 is the principal transporter for the transfer of glucose between the liver and blood, and renal glucose reabsorption. GLUT4 is frequently expressed in skeletal muscle, cardiac muscle and adipose tissue, the major tissues of the body that respond to insulin. GLUT4 is a major mediator of glucose removal from the circulation and functions as a key regulator of whole-body glucose homeostasis (44). It has been confirmed that increases in GLUT4 protein expression improve glucose homeostasis in dilated cardiomyopathy animals (45). Studies had been found that insulin resistance is related to a decrease in GLUT4 expression (46). It has been reported that liraglutide increases insulin secretion and target organ sensitivity to insulin, thus improving the insulin resistance state $(18,47)$. We obtained similar results, showing that liraglutide improves the glucose tolerance and insulin tolerance, decreases the value of HOMA-IR and exerts a potent protective effect on insulin resistance. However, to our knowledge, there are no reports to date as to whether liraglutide influences the gene expression of the insulin pathway in KKAy mice. Our real-time PCR results revealed that the gene expression of InsR, PI3K, GLUT2 and GLUT4 was downregulated in the diabetic KKAy mice, compared with the control C57 mice. In addition, to our knowledge, we demonstrate for the first time that liraglutide significantly increases the gene expression of GLUT4 in KKAy mouse livers. We also found that liraglutide significantly increased the expression of GLUT4 in KKAy mouse liver tissue and skeletal muscle, which was consistent with the real time-PCR results. Thus, the upregulation of GLUT4 may be one of the mechanisms involved in the effects of liraglutide in increasing insulin sensitivity and improving insulin resistance.

In conclusion, the results from the present study suggest that liraglutide ameliorates glycometabolism by increasing glycogenesis production and glycolysis, improving $\beta$ cell dysfunction and increasing insulin secretion, ameliorating insulin sensitivity and reducing insulin resistance, which may be associated with the upregulation of GLUT4. Thus, liraglutide exerts potent anti-diabetic effects. Our data provide the molecular basis for further investigation into the mechanisms by which liraglutide moderates glucose metabolism.

\section{Acknowledgements}

The present study was supported by grants from the National Natural Science Youth Foundation of China (no. 30901808) and the Technology and Innovation Project of Shaanxi Province (no. 2011KTCL03-20).

\section{References}

1. Wild S, Roglic G, Green A, Sicree R and King H: Global prevalence of diabetes: estimates for the year 2000 and projections for 2030. Diabetes Care 27: 1047-1053, 2004

2. Geraldes P and King GL: Activation of protein kinase C isoforms and its impact on diabetic complications. Circ Res 106: 1319-1331, 2010.

3. Abdin AA, Baalash AA and Hamooda HE: Effects of rosiglitazone and aspirin on experimental model of induced type 2 diabetes in rats: focus on insulin resistance and inflammatory markers. J Diabetes Complications 24: 168-178, 2010. 
4. Hayden MR and Sowers JR: Treating hypertension while protecting the vulnerable islet in the cardiometabolic syndrome. J Am Soc Hypertens 2: 239-266, 2008.

5. Li L, Miao Z, Liu R, Yang M, Liu H and Yang G: Liraglutide prevents hypoadiponectinemia-induced insulin resistance and alterations of gene expression involved in glucose and lipid metabolism. Mol Med 17: 1168-1178, 2011.

6. Mundil D, Cameron-Vendrig A and Husain M: GLP-1 receptor agonists: a clinical perspective on cardiovascular effects. Diab Vasc Dis Res 9: 95-108, 2012.

7. Senda M, Ogawa S, Nako K, Okamura M, Sakamoto T and Ito S: The glucagon-like peptide-1 analog liraglutide suppresses ghrelin and controls diabetes in a patient with Prader-Willi syndrome. Endocr J 59: 889-894, 2012.

8. Hunter K and Hölscher C: Drugs developed to treat diabetes, liraglutide and lixisenatide, cross the blood brain barrier and enhance neurogenesis. BMC Neurosci 13: 33, 2012.

9. Kesavadev J, Shankar A, Krishnan G and Jothydev S: Liraglutide therapy beyond glycemic control: an observational study in Indian patients with type 2 diabetes in real world setting. Int J Gen Med 5: 317-322, 2012.

10. Fujishima Y, Maeda N, Inoue K, et al: Efficacy of liraglutide, a glucagon-like peptide-1 (GLP-1) analogue, on body weight, eating behavior, and glycemic control, in Japanese obese type 2 diabetes. Cardiovasc Diabetol 11: 107, 2012.

11. Fukushima T, Arai T, Ariga-Nedachi M, et al: Insulin receptor substrates form high-molecular-mass complexes that modulate their availability to insulin/insulin-like growth factor-I receptor tyrosine kinases. Biochem Biophys Res Commun 404: 767-773, 2011.

12. Zhu Y, Pereira RO, O'Neill BT, et al: Cardiac PI3K-Akt impairs insulin-stimulated glucose uptake independent of mTORC1 and GLUT4 translocation. Mol Endocrinol 27: 172-184, 2013.

13. Chen S, Wasserman DH, MacKintosh $C$ and Sakamoto K: Mice with AS160/TBC1D4-Thr649Ala knockin mutation are glucose intolerant with reduced insulin sensitivity and altered GLUT4 trafficking. Cell Metab 13: 68-79, 2011.

14. Munkonda MN, Lapointe M, Miegueu P, et al: Recombinant acylation stimulating protein administration to $\mathrm{C} 3^{-/}$mice increases insulin resistance via adipocyte inflammatory mechanisms. PLoS One 7: e46883, 2012.

15. Wei X, Wang D, Yang Y, et al: Cyanidin-3-O- $\beta$-glucoside improves obesity and triglyceride metabolism in KK-Ay mice by regulating lipoprotein lipase activity. J Sci Food Agric 91: 1006-1013, 2011.

16. Li PP, Shan S, Chen YT, et al: The PPARalpha/gamma dual agonist chiglitazar improves insulin resistance and dyslipidemia in MSG obese rats. Br J Pharmacol 148: 610-618, 2006.

17. Drucker DJ: The biology of incretin hormones. Cell Metab 3: 153-165, 2006.

18. Porter DW, Kerr BD, Flatt PR, Holscher C and Gault VA: Four weeks administration of Liraglutide improves memory and learning as well as glycaemic control in mice with high fat dietary-induced obesity and insulin resistance. Diabetes Obes Metab 12: 891-899, 2010.

19. Gault VA, Kerr BD, Harriott $P$ and Flatt PR: Administration of an acylated GLP-1 and GIP preparation provides added beneficial glucose-lowering and insulinotropic actions over single incretins in mice with type 2 diabetes and obesity. Clin Sci (Lond) 121 107-117, 2011.

20. Shimoda M, Kanda Y, Hamamoto S, et al: The human glucagon-like peptide-1 analogue liraglutide preserves pancreatic beta cells via regulation of cell kinetics and suppression of oxidative and endoplasmic reticulum stress in a mouse model of diabetes. Diabetologia 54: 1098-1108, 2011.

21. MagnoniLJ, Vraskou Y,Palstra AP and Planas JV: AMP-activated protein kinase plays an important evolutionary conserved role in the regulation of glucose metabolism in fish skeletal muscle cells. PLoS One 7: e31219, 2012.

22. Oosterveer MH, Mataki C, Yamamoto H, et al: LRH-1-dependent glucose sensing determines intermediary metabolism in liver. $\mathrm{J}$ Clin Invest 122: 2817-2826, 2012.

23. Ros S, García-Rocha M, Calbó J and Guinovart JJ: Restoration of hepatic glycogen deposition reduces hyperglycaemia, hyperphagia and gluconeogenic enzymes in a streptozotocin-induced model of diabetes in rats. Diabetologia 54: 2639-2648, 2011.
24. John S, Weiss JN and Ribalet B: Subcellular localization of hexokinases I and II directs the metabolic fate of glucose. PLoS One 6: e17674, 2011.

25. Sun L, Shukair S, Naik TJ, Moazed F and Ardehali H: Glucose phosphorylation and mitochondrial binding are required for the protective effects of hexokinases I and II. Mol Cell Biol 28: 1007-1017, 2008.

26. Holyoak T, Zhang B, Deng J, Tang Q, Prasannan CB and Fenton AW: Energetic coupling between an oxidizable cysteine and the phosphorylatable N-terminus of human liver pyruvate kinase. Biochemistry 52: 466-476, 2013.

27. Cortés-Cros M, Hemmerlin C, Ferretti S, et al: M2 isoform of pyruvate kinase is dispensable for tumor maintenance and growth. Proc Natl Acad Sci USA 110: 489-494, 2013.

28. Sun Q, Chen X, Ma J, et al: Mammalian target of rapamycin up-regulation of pyruvate kinase isoenzyme type M2 is critical for aerobic glycolysis and tumor growth. Proc Natl Acad Sci USA 108: 4129-4134, 2011.

29. Ye J, Mancuso A, Tong X, et al: Pyruvate kinase M2 promotes de novo serine synthesis to sustain mTORC1 activity and cell proliferation. Proc Natl Acad Sci USA 109: 6904-6909, 2012

30. Shirakawa J, Tanami R, Togashi Y, et al: Effects of liraglutide on beta-cell-specific glucokinase-deficient neonatal mice. Endocrinology 153: 3066-3075, 2012.

31. Janghorbani $M$ and Amini M: Incidence of metabolic syndrome and its risk factors among type 2 diabetes clinic attenders in Isfahan, Iran. Endokrynol Pol 63: 372-380, 2012.

32. Ohshima K, Mogi M, Jing F, et al: Direct angiotensin II type 2 receptor stimulation ameliorates insulin resistance in type 2 diabetes mice with PPAR $\gamma$ activation. PLoS One 7: e48387, 2012.

33. Rosengren AH, Braun M, Mahdi T, et al: Reduced insulin exocytosis in human pancreatic $\beta$-cells with gene variants linked to type 2 diabetes. Diabetes 61: 1726-1733, 2012.

34. Tersey SA, Nishiki Y, Templin AT, et al: Islet $\beta$-cell endoplasmic reticulum stress precedes the onset of type 1 diabetes in the nonobese diabetic mouse model. Diabetes 61: 818-827, 2012.

35. Emamaullee JA, Merani S, Toso C, et al: Porcine marginal mass islet autografts resist metabolic failure over time and are enhanced by early treatment with liraglutide. Endocrinology 150: 2145-2152, 2009.

36. Sturis J, Gotfredsen CF, Rømer J, et al: GLP-1 derivative liraglutide in rats with beta-cell deficiencies: influence of metabolic state on beta-cell mass dynamics. Br J Pharmacol 140: 123-132, 2003.

37. Knudsen LB: Liraglutide: the therapeutic promise from animal models. Int J Clin Pract 167: 4-11, 2010.

38. Kawamori R: Insulin resistance seen in non-insulin dependent diabetes mellitus and hypertension. Hypertens Res 19 Suppl 1: S61-S64, 1996.

39. Tomimoto S, Ojika T, Shintani N, et al: Markedly reduced white adipose tissue and increased insulin sensitivity in adcyap1-deficient mice. J Pharmacol Sci 107: 41-48, 2008.

40. Taniguchi CM, Emanuelli B and Kahn CR: Critical nodes in signalling pathways: insights into insulin action. Nat Rev Mol Cell Biol 7: 85-96, 2006.

41. Inokuchi J: Membrane microdomains and insulin resistance. Febs Lett 584: 1864-1871, 2010.

42. Chang YC and Chuang LM: The role of oxidative stress in the pathogenesis of type 2 diabetes: from molecular mechanism to clinical implication. Am J Transl Res 2: 316-331, 2010.

43. Muniyappa R, Montagnani M, Koh KK and Quon MJ: Cardiovascular actions of insulin. Endocr Rev 28: 463-491, 2007.

44. Lee JO, Song YH, Kim MW, et al: A sub-1-volt nanoelectromechanical switching device. Nat Nanotechnol 8: 36-40, 2013.

45. Giannocco G, Oliveira KC, Crajoinas RO, et al: Dipeptidyl peptidase IV inhibition upregulates GLUT4 translocation and expression in heart and skeletal muscle of spontaneously hypertensive rats. Eur J Pharmacol 698: 74-86, 2013.

46. Nieto-Vazquez I, Fernández-Veledo S, de Alvaro $C$ and Lorenzo M: Dual role of interleukin-6 in regulating insulin sensitivity in murine skeletal muscle. Diabetes 57: 3211-3221, 2008.

47. Zhang WY, Lee JJ, Kim IS, Kim Y, Park JS and Myung CS: 7-O-methylaromadendrin stimulates glucose uptake and improves insulin resistance in vitro. Biol Pharm Bull 33: 1494-1499, 2010. 